\title{
Round Complexity of Authenticated Broadcast with a Dishonest Majority*
}

\author{
Juan A. Garay ${ }^{\dagger} \quad$ Jonathan Katz ${ }^{\ddagger}$ Chiu-Yuen $\mathrm{Koo}^{\S} \quad$ Rafail Ostrovsky \\ Appeared in FOCS 2007: 658-668
}

\begin{abstract}
Broadcast among $n$ parties in the presence of $t \geq n / 3$ malicious parties is possible only with some additional setup. The most common setup considered is the existence of a PKI and secure digital signatures, where so-called authenticated broadcast is achievable for any $t<n$.

It is known that $t+1$ rounds are necessary and sufficient for deterministic protocols achieving authenticated broadcast. Recently, however, randomized protocols running in expected constant rounds have been shown for the case of $t<n / 2$. It has remained open whether randomization can improve the round complexity when an honest majority is not present. We address this question and show upper/ lower bounds on how much randomization can help:

- For $t \leq n / 2+k$, we show a randomized broadcast protocol that runs in expected $\mathcal{O}\left(k^{2}\right)$ rounds. In particular, we obtain expected constant-round protocols for $t=n / 2+\mathcal{O}(1)$.

- On the negative side, we show that even randomized protocols require $\Omega(2 n /(n-t))$ rounds. This in particular rules out expected constant-round protocols when the fraction of honest parties is sub-constant.
\end{abstract}

\footnotetext{
${ }^{*}$ A portion of this work was done while the authors were visiting the Institute for Pure and Applied Mathematics (IPAM), UCLA.

${ }^{\dagger}$ Bell Labs, Alcatel-Lucent. E-mail: garay@research.bell-labs.com.

${ }_{\ddagger}^{\ddagger}$ Dept. of Computer Science, University of Maryland. E-mail: $\{j k a t z$, cykoo\}@ cs . umd. edu. Work of J.K. supported in part by NSF CAREER award \#0447075 and US-Israel Binational Science Foundation grant \#2004240.

${ }^{\S}$ Dept. of Computer Science, University of Maryland, E-mail: cykoo@cs . umd. edu .

IDepts. of Computer Science and Mathematics, UCLA. E-mail: rafailecs.ucla.edu. Work supported in part by an IBM Faculty Award, a Xerox Innovation Group Award, NSF Cybertrust grant \#0430254, and a U.C. MICRO grant.
} 


\section{Introduction}

Designing protocols for simulating a broadcast channel over a point-to-point network in the presence of faults is a fundamental problem in distributed computing and cryptography. Much work has focused both on characterizing the feasibility of protocols for solving the problem in different settings, as well as on the inherent round complexity of such protocols. In a synchronous network with pairwise authenticated channels and no additional setup, the classical results of Pease, Shostak, and Lamport $[24,29]$ show that broadcast among $n$ parties is achievable if and only if the number of malicious parties $t$ satisfies $t<n / 3$. In this setting, a lower bound of $t+1$ rounds for any deterministic protocol is known [16]. A protocol with this round complexity - but with exponential message complexity — was shown in the initial work by Pease et al. [24, 29]. Following a long sequence of works [9, 1, 33, 12, 26, 5, 4], Garay and Moses [19] showed a deterministic, polynomial-time Byzantine agreement protocol having optimal resilience $t<n / 3$ and optimal round complexity $t+1$.

To circumvent the above-mentioned lower bound on the round complexity (as well as impossibility results for asynchronous networks [15]), researchers beginning with Rabin [31] and Ben-Or [2] explored the use of randomization. (See [7] for an early survey on the subject.) This culminated in the work of Feldman and Micali [14], who showed a broadcast protocol with optimal resilience that runs in expected constant rounds. ${ }^{1}$

To achieve resilience $t \geq n / 3$, additional assumptions are needed even if randomization is used. The most common assumptions are the existence of digital signatures and the presence of a public-key infrastructure (PKI) established among the $n$ parties in the network; this is referred to as the authenticated setting. Pease et al. [29, 24] showed an authenticated broadcast protocol for any $t<n$, and a polynomial-time protocol achieving this resilience was given by Dolev and Strong [13].

The $(t+1)$-round lower bound for deterministic protocols holds in the authenticated setting as well [13], and the known protocols [29, 24, 13] meet this bound. Randomized protocols running in expected constant rounds for $t<n / 2$ have been shown by Fitzi and Garay [17] (based on [6, 28]) under specific number-theoretic assumptions, and by Katz and Koo [23] based on signatures and a PKI alone.

When an honest majority is not available (i.e., $t \geq n / 2$ ), there has been no progress since the initial work of $[29,24,13]$ on improving the round complexity of authenticated broadcast. ${ }^{2}$ Besides being an interesting and fundamental problem in its own right, authenticated broadcast is often used as a sub-routine within larger protocols that are designed and analyzed using the abstraction that a broadcast channel exists. For the specific case of secure multi-party computation with a dishonest majority, we remark that although meaningful security notions can be achieved even without broadcast [20], and fairness cannot be achieved even with broadcast [8], there are still advantages to having broadcast available. Specifically, broadcast can be used to achieve unanimous abort [20], or partial notions of fairness [18, 22]. In contrast, the constant-round "broadcast-with-

\footnotetext{
${ }^{1}$ The Feldman-Micali protocol requires private channels. Goldwasser et al. [21] show a broadcast protocol for $t \leq n /(3+\epsilon)$ that runs in expected $\mathcal{O}(\log n)$ rounds and does not require private channels.

${ }^{2}$ The techniques used for $t<n / 2$ do not immediately translate to the case of $t \geq n / 2$ : a key building block in the former setting is verifiable secret sharing, which is not even feasible in the latter setting.
} 
abort" protocol of [20] does not appear to suffice for such applications.

Our contributions. In this paper we make the first progress toward characterizing when randomized protocols can beat the $(t+1)$-round barrier for $t \geq n / 2$.

- We show a randomized broadcast protocol tolerating $t \leq n / 2+k$ malicious parties that terminates in an expected $\mathcal{O}\left(k^{2}\right)$ rounds. This is an improvement over existing state of the art for $t=n / 2+o(\sqrt{n})$, and gives an expected constant-round protocol when $t=n / 2+\mathcal{O}(1)$.

- We show that no randomized broadcast protocol tolerating $t$ malicious parties terminates in $2 n /(n-t)-2$ or fewer rounds. This in particular means that when the fraction of honest parties is sub-constant, it is impossible to obtain protocols with expected constant round complexity. It also implies that the Dolev-Strong protocol [13] has optimal round complexity (to within a constant factor) when $t=n-\mathcal{O}(1)$.

Organization. In Section 2.1, we describe our model and give the standard definitions of broadcast and Byzantine agreement. We present the technical tools we use in Section 2.2; these include a generalization of gradecast [14] that may be of independent interest. We present our new broadcast protocol in Section 3, and prove our impossibility result in Section 4. Some proofs are deferred to the Appendix.

\section{Preliminaries}

\subsection{Model and Definitions}

We assume a standard point-to-point network in which parties $P_{1}, P_{2}, \ldots, P_{n}$ communicate in synchronous rounds using pairwise private and authenticated channels. When we say a protocol tolerates $t$ dishonest parties, we always mean that it is secure against a rushing adversary who may adaptively corrupt up to $t$ parties during execution of the protocol and coordinate the actions of these parties as they deviate from the protocol in an arbitrary manner. ${ }^{3}$ Parties not corrupted by the adversary are called honest.

The existence of a PKI means that prior to execution of the protocol all parties hold the same vector $\left(p k_{1}, \ldots, p k_{n}\right)$ of public keys for a digital signature scheme, and each honest party $P_{i}$ holds the honestly generated secret key $s k_{i}$ associated with $p k_{i}$. When we describe signature computation in our protocols, we omit for simplicity certain additional information that should be signed along with the message. That is, when we say that party $P_{i}$ signs message $m$ and sends it to $P_{j}$, we implicitly mean that $P_{i}$ signs the concatenation of $m$ with additional information such as: (1) the identity of the recipient $P_{j}$, (2) the current round number, (3) an identifier for the message (in case multiple messages are sent to $P_{j}$ in the same round); and (4) an identifier for the particular (sub-)protocol to which $m$ belongs (in case multiple sub-protocols are being run; cf. [25]). This information is also verified, as appropriate, when the signature is verified.

\footnotetext{
${ }^{3} \mathrm{~A}$ rushing adversary waits until it receives messages from all honest parties in a given round before sending any messages of its own for that round. Adaptive corruption means that the adversary is allowed to corrupt parties on the fly, as opposed to deciding which parties to corrupt before execution of the protocol begins.
} 
We assume in our proofs that the adversary cannot forge valid signatures on behalf of honest parties. Using a standard hybrid argument and assuming the existence of one-way functions [27, 32], this implies that our protocols are secure against any computationally-bounded adversary. (Alternately, if stronger setup is assumed then information-theoretic pseudo-signatures [30] can be used.)

We now give the standard definition of broadcast [24].

Definition 1 (Broadcast). A protocol for parties $\mathcal{P}=\left\{P_{1}, \ldots, P_{n}\right\}$, where a distinguished sender $P^{*} \in \mathcal{P}$ holds an initial input $m$, is a broadcast protocol tolerating $t$ malicious parties if the following conditions hold for any adversary controlling at most t parties:

Agreement: All honest parties output the same value.

Validity: If the sender is honest, then all honest parties output $m$.

We will also rely on protocols for the related task of Byzantine agreement (BA). Here, each party holds an initial input: the agreement condition remains the same as above; validity requires that if all honest parties hold initial input $m$, then all honest parties will output $m$. Note that BA is impossible to achieve for $t \geq n / 2$ (in any setting).

\subsection{Tools}

We describe two technical tools we use to construct our randomized broadcast protocol.

BA in expected constant rounds for $t<n / 2$. The work of Katz and Koo [23] gives an authenticated BA protocol $\mathrm{BA}_{\text {HonestMaj }}$ tolerating any $t<n / 2$ malicious parties and running in expected constant rounds. Protocol $\mathrm{BA}_{\text {HonestMaj }}$ satisfies the following stronger property that we will rely on in the present work:

Lemma 1. If $h>n / 2$ honest parties start $\mathrm{BA}_{\mathrm{HonestMaj}}$ with the same input, then all honest parties terminate protocol $\mathrm{BA}_{\mathrm{HonestMaj}}$ in exactly $K$ rounds for some constant $K$.

Gradecast. Gradecast, a generalization of crusader agreement [11], was introduced by Feldman and Micali [14]. As opposed to broadcast, where the honest parties are required to reach a unanimous decision, in gradecast the honest parties are allowed to disagree by "a small amount". Specifically, parties now output a grade along with their output value; the grade output by a party can be viewed as the "confidence" of this party in the sender. The gradecast protocol given by Feldman and Micali supports the three grades $\{0,1,2\}$, and runs in three rounds. Here, we generalize their protocol to the case of an arbitrary number of grades. We first present the definition:

Definition 2 (Gradecast with multiple grades). A protocol for parties $\mathcal{P}=\left\{P_{1}, \ldots, P_{n}\right\}$, where $P^{*} \in \mathcal{P}$ holds an initial input $m$, is a $g^{*}$-gradecast protocol (tolerating $n-1$ malicious parties) if the following conditions hold for any adversary controlling any number of parties:

Functionality: An honest party $P_{i}$ outputs a message $m_{i}$ and a grade $g_{i} \in\left\{0,1, \ldots, g^{*}\right\}$.

Correctness: If the sender is honest, then $m_{i}=m$ and $g_{i}=g^{*}$ for all honest parties $P_{i}$. 
Soundness: Let $P_{i}, P_{j}$ be any two honest parties. If $g_{i} \geq 2$, then $m_{j}=m_{i}$ and $g_{j} \geq g_{i}-1$. If $g_{i}=1$, then $m_{j}=m_{i}$ or $g_{j}=0$.

A similar primitive called "proxcast" was defined and constructed by Considine et al. [10]. Our construction differs from theirs in two ways. First, our construction is in the authenticated setting while theirs relies on the existence of " $k$-cast channels". Second, our protocol can tolerate any number of dishonest parties, while theirs only tolerates a constant fraction (the exact constant depends on the value of $k$ ) of malicious participants.

We now demonstrate a construction of $g^{*}$-gradecast for any value $g^{*}$. Specifically, we define a protocol M-Gradecast $\left(m, g^{*}\right)$ where $m$ represents the initial value of the sender and $g^{*}$ denotes the maximum supported grade. In the description that follows, each party $P_{i}$ starts with internal variables $\bar{g}_{i}, S_{i}$, and $m_{i}$ initialized to 0 , the empty set, and $\perp$, respectively.

Protocol M-Gradecast $\left(m, g^{*}\right)$

Round 1: The sender computes a signature $\sigma$ on $m$ and sends $(m, \sigma)$ to all parties.

Round 2 to Round $2 g^{*}+1$ :

Step (a) Each party $P_{i}$ does as follows: For each tuple $\left(m^{\prime}, \sigma^{\prime}\right)$ received by the end of the previous round, if $\sigma^{\prime}$ is a valid signature by the sender on $m^{\prime}$ and $m^{\prime} \notin S_{i}$, then:

- Set $S_{i}:=S_{i} \cup\left\{m^{\prime}\right\}$. If $\left|S_{i}\right|=1$, then set $m_{i}:=m^{\prime}$.

- $P_{i}$ sends $\left(m^{\prime}, \sigma^{\prime}\right)$ to all other parties.

Step (b) If $\left(m_{i} \neq \perp\right)$ and $\left(\left|S_{i}\right|=1\right)$ then set $\bar{g}_{i}:=\bar{g}_{i}+1$.

Output determination: Each party $P_{i}$ sets $g_{i}:=\left\lfloor\bar{g}_{i} / 2\right\rfloor$ and outputs $\left(m_{i}, g_{i}\right)$.

Lemma 2. Protocol M-Gradecast $\left(\cdot, g^{*}\right)$ is a $g^{*}$-gradecast protocol with round complexity $2 g^{*}+1$.

The proof is given in the Appendix.

\section{Randomized Broadcast Protocols for Dishonest Majority}

As a warm-up, we first construct an expected constant-round broadcast protocol for the special case of $t=n / 2$ (and $n$ even) before dealing with the more general case.

\subsection{The Case $t=n / 2$}

The main idea here is as follows: in the first phase, the sender will gradecast its input $m$. If the sender is honest, this gradecast is already enough to implement broadcast; on the other hand, if the other parties catch the sender cheating then they can exclude the sender and determine their output by executing $\mathrm{BA}_{\text {HonestMaj }}$. The key point is that in the latter case, assuming $t=n / 2$ to begin with, an honest majority is present once the dishonest dealer is excluded. (Variants of this idea - i.e., executing a protocol until either something good happens or some dishonest parties can be excluded - have been used in prior work on Byzantine agreement $[1,26,5,19]$.) Of course, we need to handle the scenario where some parties believe the sender is honest while other parties 
catch the sender cheating; this can be done using the grades obtained in the initial gradecast. We now provide a formal description of the protocol:

Phase I $P^{*}$, who holds input $m$, acts as the sender in an execution of M-Gradecast $(m, 2)$, outputs $m$, and then exits the protocol. Let $\left(m_{i}, g_{i}\right)$ denote the output of $P_{i}$ in this step.

Phase II All parties except $P^{*}$ (who has already exited the protocol) run $\mathrm{BA}_{\text {HonestMaj }}$ in the following way:

- If $g_{i}=2$, then $P_{i}$ enters protocol $\mathrm{BA}_{\text {HonestMaj }}$ with input $m_{i}$, terminates $\mathrm{BA}_{\text {HonestMaj }}$ after $K$ rounds (where $K$ is the constant from Lemma 1), and outputs $m_{i}$. We stress that $P_{i}$ outputs $m_{i}$ regardless of the output (if any) of protocol $\mathrm{BA}_{\mathrm{HonestMaj}}$.

- Otherwise (i.e., $g_{i}<2$ ), $P_{i}$ enters protocol BA HonestMaj with input $m_{i}$, runs $\mathrm{BA}_{\text {HonestMaj }}$ until successful termination of the protocol, and outputs whatever directed to by $\mathrm{BA}_{\text {HonestMaj }}$.

We now argue that the above protocol achieves broadcast for $t=n / 2$ in expected constant rounds. If the sender is honest then, by the correctness property of M-Gradecast $(m, 2)$, each honest party $P_{i}$ outputs $\left(m_{i}=m, g_{i}=2\right)$ in Phase I and thus, in Phase II, outputs $m_{i}=m$ after executing $\mathrm{BA}_{\text {HonestMaj }}$ for exactly $K$ rounds. As the round complexity of Phase I is constant, the entire protocol runs for a strict constant number of rounds.

If the sender is dishonest, then protocol $\mathrm{BA}_{\text {HonestMaj }}$ is run with an honest majority. There are two sub-cases to consider. The first sub-case is that there exists an honest party $P_{i}$ whose output in Phase $\mathrm{I}$ is $\left(m_{i}, g_{i}=2\right)$. Then by the soundness property of M-Gradecast $(m, 2)$, all honest parties

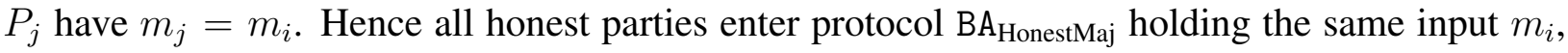
and the protocol $\mathrm{BA}_{\text {HonestMaj }}$ terminates after $K$ rounds with each honest party $P_{j}$ outputting $m_{i}$, regardless of the grade $g_{j}$ it output in the first step. The second sub-case is when all honest parties output a grade less than 2 in Phase I. Then all honest parties run $\mathrm{BA}_{\mathrm{HonestMaj}}$ until termination, and so all honest parties output the same value in expected constant rounds.

\subsection{The Case $t \leq n / 2+k$}

In this section we construct a broadcast protocol Rand-Bcast for $t \leq n / 2+k$ that runs in expected $\mathcal{O}\left(k^{2}\right)$ rounds. For simplicity, we assume $n$ is even and so $t=n / 2+k$. (Everything that follows works also for $n$ odd, though things can be optimized somewhat.) Set $c \stackrel{\text { def }}{=} 2 k$; this is equal to the difference between the number of dishonest parties and the number of honest parties. Without loss of generality, let $P_{1}$ be the sender. Rand-Bcast consists of two phases: Phase I takes exactly $\mathcal{O}\left(c^{2}\right)$ rounds, while Phase II runs for $\mathcal{O}(1)$ rounds in expectation. At the end of Phase I, each party in Init $\stackrel{\text { def }}{=}\left\{P_{1}, \ldots, P_{c+1}\right\}$ outputs a message, which will be its final output for the entire protocol, while each party $P_{i}$ in Rem $\stackrel{\text { def }}{=}\left\{P_{c+2}, \ldots, P_{n}\right\}$ outputs a tuple of the form $\left\{\left(m_{i, 1}, g_{i, 1}\right)\right.$, $\left.\left(m_{i, 2}, g_{i, 2}\right), \ldots,\left(m_{i, c+1}, g_{i, c+1}\right)\right\}$. In the second phase, parties in Rem $=\left\{P_{c+2}, \ldots, P_{n}\right\}$ determine their outputs using the output they obtained in Phase I. Parties in Init $=\left\{P_{1}, \ldots, P_{c+1}\right\}$ do not take part in Phase II.

Phase I is based on the authenticated broadcast protocol of Dolev and Strong [13] which tolerates any $t<n$ dishonest parties and has the property that, in each round, honest parties send the 
same message to all other parties. Roughly speaking, parties $P_{1}, \ldots, P_{c+1}$ will execute the DolevStrong protocol with the following twist: whenever a party $P_{i}$ (in the Dolev-Strong protocol) is supposed to send a message to every other party in $\left\{P_{1}, \ldots, P_{c+1}\right\}$, party $P_{i}$ instead gradecasts the message to all $n$ parties in the network using protocol M-Gradecast from Section 2.2. This has the effect of allowing parties $P_{c+2}, \ldots, P_{n}$ to "monitor" the execution of the Dolev-Strong protocol being run by parties $P_{1}, \ldots, P_{c+1}$.

The Dolev-Strong protocol guarantees that broadcast is achieved among $P_{1}, \ldots, P_{c+1}$ at the end of Phase I. As mentioned earlier, each remaining party $P_{i} \in\left\{P_{c+2}, \ldots, P_{n}\right\}$ outputs $\left\{\left(m_{i, 1}, g_{i, 1}\right)\right.$, $\left.\left(m_{i, 2}, g_{i, 2}\right), \ldots,\left(m_{i, c+1}, g_{i, c+1}\right)\right\}$ based on the messages and grades it received in Phase I. Informally, $m_{i, k}$ is the message that $P_{i}$ "believes" $P_{k}$ will output, with $g_{i, k}$ indicating the level of "confidence" $P_{i}$ has in this determination. In particular, if $P_{k}$ is honest then $m_{i, k}$ will be equal to the message output by $P_{k}$ and $g_{i, k}$ will be the maximum possible grade. Furthermore, based on the properties of M-Gradecast, a relaxed form of agreement is achieved among the remaining parties. Specifically, for any honest parties $P_{i}, P_{j} \in\left\{P_{c+2}, \ldots, P_{n}\right\}$ and $k \in\{1, \ldots, c+1\}$ we have:

- If $g_{i, k}>1$, then $m_{i, k}=m_{j, k}$ and $g_{j, k} \geq g_{i, k}-1$.

- If $g_{i, k}=1$, then $m_{i, k}=m_{j, k}$ or $g_{j, k}=0$.

Therefore, although the remaining honest parties may not reach a unanimous decision when $P_{k}$ is dishonest, the remaining honest parties will only disagree by "a small amount".

In Phase II, each remaining party $P_{i}$ first locally "combines" its output $\left\{\left(m_{i, 1}, g_{i, 1}\right),\left(m_{i, 2}, g_{i, 2}\right)\right.$, $\left.\ldots,\left(m_{i, c+1}, g_{i, c+1}\right)\right\}$ into a single message/grade pair $\left(m_{i}, g_{i}\right)$, with $g_{i} \in\{0,1,2\}$, such that the following hold for all honest parties $P_{i}, P_{j} \in\left\{P_{c+2}, \ldots, P_{n}\right\}$ :

- If there exists an honest party $P_{k} \in\left\{P_{1}, \ldots, P_{c+1}\right\}$, then $m_{i}$ is equal to the message output by $P_{k}$, and $g_{i}=2$ (the maximum possible grade).

- If $g_{i}=2$, then $m_{i}=m_{j}$ and $g_{j} \geq 1$.

Finally, parties $P_{c+2}, \ldots, P_{n}$ determine their final output as in Phase II of the broadcast protocol for $t=n / 2$ described earlier. The key observation is that if there exists even a single honest party $P_{k} \in\left\{P_{1}, \ldots, P_{c+1}\right\}$, then for every honest party $P_{i} \in\left\{P_{c+2}, \ldots, P_{n}\right\}$ it holds that $m_{i}=m_{k}$ (where $m_{k}$ is the output of $P_{k}$ ) and $g_{i}=2$; otherwise (i.e., if $P_{1}, \ldots, P_{c+1}$ are all dishonest), a majority of the remaining parties are honest, and so they can rely on the output of $B A_{\text {HonestMaj }}$.

Gradecast is also used as a building block in the (expected) sub-linear broadcast protocols of $[14,23,3,21]$. In these works, gradecast is used to replace the broadcast channel in various subprotocols that are run among all $n$ parties in the network; these sub-protocols achieve some relaxed functionality that suffices for achieving broadcast. Here, we use gradecast in a different way, by having a small subset of the parties run some sub-protocol while gradecasting their messages to all parties in the network.

We now describe the two phases of the protocol in more detail, and prove the protocol's correctness. 


\subsubsection{Phase I}

Set $g^{*} \stackrel{\text { def }}{=} 2^{\lceil\log (c+1)\rceil+1}+2^{\lceil\log (c+1)\rceil}-1 .^{4}$ Recall that we assume, without loss of generality, that $P_{1}$ is the sender. Let Init $\stackrel{\text { def }}{=}\left\{P_{1}, \ldots, P_{c+1}\right\}$ (these are the parties who run the Dolev-Strong protocol in the initial phase) and let Rem $\stackrel{\text { def }}{=}\left\{P_{c+2}, \ldots, P_{n}\right\}$ (these are the parties who remain in the second phase). Each party $P_{i} \in$ Init $\backslash\left\{P_{1}\right\}$ has a variable $M_{i}$ initialized to the empty set; each party $P_{i} \in$ Rem has variables $g_{i, 1}, \ldots, g_{i, c+1}$ all initialized to $g^{*}$, and variables $M_{i, 1}, \ldots, M_{i, c+1}$ all initialized to the empty set.

Roughly speaking, when a party $P_{i} \in$ Init $\backslash\left\{P_{1}\right\}$ receives a new message that originated from $P_{1}$ (with correct signatures attached), then as long as $\left|M_{i}\right|<2$ it signs and gradecasts the received message, and adds the message to $M_{i}$. However, $P_{i}$ stops adding new messages once $\left|M_{i}\right|=2$, as this means $P_{i}$ has received valid signatures of the sender on two different messages (and so $P_{i}$ knows the sender is dishonest). Each $P_{i}$ determines its output based on the contents of $M_{i}$ at the end of Phase I.

Each party $P_{i} \in$ Rem acts as follows: every time it hears $P_{j} \in$ Init gradecast a new message that originated from $P_{1}$ (with correct signatures attached), then as long as $\left|M_{i, j}\right|<2$ it adds the message to $M_{i, j}$ and updates $g_{i, j}$ based on the grade it received in the aforementioned execution of gradecast. At the end of Phase I, $P_{i}$ determines $M_{i, j}$ (i.e., its determination as to what $P_{j}$ will output) based on the contents of $M_{i, j}$.

Protocol Rand-Bcast - Phase I

Step 1: $P_{1}$ computes a signature $\sigma$ of $m$, runs M-Gradecast $\left(\left(m, \sigma, P_{1}\right), g^{*}\right)$, outputs $m$, and exits the protocol.

Step $j$, for $2 \leq j \leq c+2$ :

1. Each $P_{i}$ does the following: For each gradecast performed in the previous step, let $\left(m_{i, \ell}^{\prime}, g_{i, \ell}^{\prime}\right)$ be the local output (of party $P_{i}$ ) of an invocation of M-Gradecast with $P_{\ell} \in \operatorname{Init}$ as the sender. (Note: each $P_{\ell}$ may gradecast multiple times in a given step. The output of each gradecast is handled separately.) Let $m_{i, \ell}^{\prime}$ have the form $\left(m, \sigma_{\alpha_{0}}, P_{1}, \sigma_{\alpha_{1}}, P_{\alpha_{1}}, \ldots, \sigma_{\alpha_{j-2}}, P_{\alpha_{j-2}}=P_{\ell}\right)$. If $P_{1}, P_{\alpha_{1}}, \ldots, P_{\alpha_{j-2}} \in$ Init are all unique; $\sigma_{\alpha_{0}}$ is a valid signature on $m$ by $P_{1}$; and $\sigma_{\alpha_{k}}$ is a valid signature on $\sigma_{\alpha_{k-1}}$ by $P_{\alpha_{k}}$ for $1 \leq k \leq j-2$ (if all these conditions hold, we say $m_{i, \ell}^{\prime}$ is valid in step $j$ ), then:

Case 1: $P_{i} \in$ Init $\backslash\left\{P_{1}\right\}$. If $j<c+2, m \notin M_{i}$ and $\left|M_{i}\right|<2$, then: set $M_{i}:=M_{i} \cup\{m\}$; compute a signature $\sigma_{\alpha_{j-1}}$ on $\sigma_{\alpha_{j-2}}$; and run M-Gradecast $\left(\left(m_{i, \ell}^{\prime}, \sigma_{\alpha_{j-1}}, P_{i}\right), g^{*}\right)$.

Case 2: $P_{i} \in \operatorname{Rem}$. Set $g_{i, \ell}:=\min \left\{g_{i, \ell}, g_{i, \ell}^{\prime}\right\}$. If $m \notin M_{i, \ell}$ and $\left|M_{i, \ell}\right|<2$, then set $M_{i, \ell}:=$ $M_{i, \ell} \cup\{m\}$.

2. If $P_{i} \in \operatorname{Init} \backslash\left\{P_{1}\right\}$ : Let $d \leq 2$ denote the number of times $P_{i}$ has already run M-Gradecast in this step. Run $2-d$ invocations of M-Gradecast('nothing', $g^{*}$ ). (This ensures that each $P_{i} \in$ Init $\backslash\left\{P_{1}\right\}$ acts as the sender in exactly two executions of M-Gradecast in each step.)

\footnotetext{
${ }^{4}$ Jumping ahead, the reason $g^{*}$ is set to this particular value is related to the second phase of the protocol. In Phase II, the parties will combine $c+1$ message/grade pairs into a single message/grade pair in a sequence of $\log (c+1)$ steps. In each step, the maximum possible grade will be reduced by half, and we set $g^{*}$ to this particular value so that the final grade will lie between 0 and 2 .
} 
Output determination: Let $\perp$ and $\phi$ be two special symbols, with $\perp$ indicating that a party has received two different messages with valid signatures of the sender, and $\phi$ indicating that a party did not receive any messages with a valid signature of the sender.

Each party $P_{i} \in$ Init $\backslash\left\{P_{1}\right\}$ does: If $\left|M_{i}\right|=2$, output $\perp$; if $\left|M_{i}\right|=1$, output the message in $M_{i}$; if $\left|M_{i}\right|=0$, output $\phi$.

Each party $P_{i} \in$ Rem does: For each $P_{\ell} \in$ Init, compute $m_{i, \ell}$ as follows:

- If $\left|M_{i, \ell}\right|=2$, set $m_{i, \ell}:=\perp$; if $\left|M_{i, \ell}\right|=1$, set $m_{i, \ell}$ to be the message in $M_{i, \ell}$; if $\left|M_{i, \ell}\right|=0$, set $m_{i, \ell}:=\phi$.

The round complexity of Phase I is $\mathcal{O}\left(k^{2}\right)$ as claimed. We now state several properties related to the first phase of our protocol (proofs appear in the Appendix). Phase II of Rand-Bcast is described in Section 3.2.2.

Lemma 3. If the sender $P_{1}$ is honest, the following holds at the end of Phase I:

\section{All honest parties in Init $\backslash\left\{P_{1}\right\}$ output $m$;}

2. For all honest parties $P_{i} \in$ Rem, it holds that $m_{i, 1}=m$ and $g_{i, 1}=g^{*}$. Furthermore, for each $2 \leq j \leq c+1$ it holds that $m_{i, j}=m$ or $m_{i, j}=\phi$ (this holds even if $P_{j}$ is dishonest).

The next three lemmas concern the case when there exists an honest party in Init $\backslash\left\{P_{1}\right\}$.

Lemma 4. If any honest party $P_{i} \in$ Init $\backslash\left\{P_{1}\right\}$ outputs $\perp$, then all honest parties in Init $\backslash\left\{P_{1}\right\}$ output $\perp$, and for any honest $P_{j} \in$ Rem it holds that $m_{j, i}=\perp$ and $g_{j, i}=g^{*}$ at the end of Phase I.

Lemma 5. If any honest party $P_{i} \in \operatorname{Init} \backslash\left\{P_{1}\right\}$ outputs $\phi$, then all honest parties in Init $\backslash\left\{P_{1}\right\}$ output $\phi$, and for any honest $P_{j} \in \operatorname{Rem}$ it holds that $m_{j, i}=\phi$ and $g_{j, i}=g^{*}$ at the end of Phase I. Moreover, if $m_{j, k} \neq \phi$ for some $k \in\{1, \ldots, c+1\}$, then $g_{j, k} \leq 1$.

Lemma 6. If any honest party $P_{i} \in$ Init $\backslash\left\{P_{1}\right\}$ outputs $m \notin\{\perp, \phi\}$, then all honest parties in Init $\backslash\left\{P_{1}\right\}$ output $m$, and for any honest $P_{j} \in \operatorname{Rem}$ it holds that $m_{j, i}=m$ and $g_{j, i}=g^{*}$ at the end of Phase I. Moreover, if $m_{j, k} \neq m$ and $m_{j, k} \neq \phi$ for some $k \in\{1, \ldots, c+1\}$, then $g_{j, k} \leq 1$.

The next lemma states that some relaxed form of agreement exists among the parties in Rem regarding their determination as to what a (dishonest) $P_{\ell} \in$ Init outputs. (Note that the case of an honest $P_{\ell}$ is handled in the previous three lemmas.) The lemma follows directly from the properties of gradecast and the specification of Phase I.

Lemma 7. For $1 \leq \ell \leq c+1$, at the end of Phase I:

- If an honest party $P_{i} \in \operatorname{Rem}$ has $g_{i, \ell}>1$, then all honest parties $P_{j} \in$ Rem have both $m_{j, \ell}=m_{i, \ell}$ and $g_{j, \ell} \geq g_{i, \ell}-1$.

- If an honest party $P_{i} \in \operatorname{Rem}$ has $g_{i, \ell}=1$, then all honest parties $P_{j} \in$ Rem have either $m_{j, \ell}=m_{i, \ell}$ or $g_{j, \ell}=0$. 


\subsubsection{Phase II}

In the second phase of the protocol, the parties in Rem determine their outputs based on the information they obtained in the first phase. Recall that by the end of Phase $\mathrm{I}$, each $P_{i}$ holds values $\left\{\left(m_{i, 1}, g_{i, 1}\right),\left(m_{i, 2}, g_{i, 2}\right), \ldots,\left(m_{i, c+1}, g_{i, c+1}\right)\right\}$ where $0 \leq g_{i, j} \leq g^{*}$ for all $1 \leq j \leq c+1$. In Phase II, based on these values, each $P_{i}$ first locally computes a single message/grade pair $\left(m_{i}^{(0)}, g_{i}^{(0)}\right)$, and then determines its output as in Phase II of the protocol for $t=n / 2$ described earlier. The message/grade $\left(m_{i}^{(0)}, g_{i}^{(0)}\right)$ is computed from $\left\{\left(m_{i, 1}, g_{i, 1}\right),\left(m_{i, 2}, g_{i, 2}\right), \ldots,\left(m_{i, c+1}, g_{i, c+1}\right)\right\}$ in a sequence of $\lceil\log (c+1)\rceil$ (non-interactive) steps: in each step the number of message/grade pairs is reduced by half by "combining" two adjacent message/grade pairs into a single pair.

Before we describe the second phase of the protocol, we first describe a subroutine which takes a value $d$, two messages $m_{1}, m_{2}$, and two grades $g_{1}, g_{2}$ (where $0 \leq g_{1}, g_{2} \leq 2^{d+1}+2^{d}-1$ ) as input, and outputs a message $m$ and a grade $g$ (where $0 \leq g \leq 2^{d}+2^{d-1}-1$ ).

Subroutine Combine $\left(d, m_{1}, m_{2}, g_{1}, g_{2}\right)$

If $\left(m_{1}=m_{2}\right)$ then

$m:=m_{1}$ and $g:=\max \left\{g_{1}-2^{d}-2^{d-1}, g_{2}-2^{d}-2^{d-1}, 0\right\} ;$

else if $\left(m_{1} \neq m_{2}\right)$ and $\left(m_{1} \neq \phi\right)$ and $\left(m_{2} \neq \perp\right)$ then

begin

If $\left(g_{1} \leq 1\right)$ and $\left(g_{2}=2^{d+1}+2^{d}-1\right)$ then $m:=m_{2}$ and $g:=2^{d}+2^{d-1}-1$

else if $\left(g_{1} \leq 2\right)$ and $\left(g_{2} \geq 2^{d+1}+2^{d}-2\right)$ then $m:=m_{2}$ and $g:=2^{d}+2^{d-1}-2$

...

else if $\left(g_{1} \leq 2^{d}+2^{d-1}\right)$ and $\left(g_{2} \geq 2^{d}+2^{d-1}\right)$ then $m:=m_{2}$ and $g:=0$

else $m:=m_{1}$ and $g:=\max \left\{g_{1}-2^{d}-2^{d-1}, 0\right\}$

end

else (Note: here, either $\left(m_{1}=\phi\right.$ and $\left.m_{2} \neq \phi\right)$ or $\left(m_{1} \neq \perp\right.$ and $\left.\left.m_{2}=\perp\right)\right)$

\section{begin}

if $\left(g_{2} \leq 1\right)$ and $\left(g_{1}=2^{d+1}+2^{d}-1\right)$ then $m:=m_{1}$ and $g:=2^{d}+2^{d-1}-1$

else if $\left(g_{2} \leq 2\right)$ and $\left(g_{1} \geq 2^{d+1}+2^{d}-2\right)$ then $m:=m_{1}$ and $g:=2^{d}+2^{d-1}-2$

‥

else if $\left(g_{2} \leq 2^{d}+2^{d-1}\right)$ and $\left(g_{1} \geq 2^{d}+2^{d-1}\right)$ then $m:=m_{1}$ and $g:=0$

else $m:=m_{2}$ and $g:=\max \left\{g_{2}-2^{d}-2^{d-1}, 0\right\}$

end

output $(m, g)$.

Each party invokes the above subroutine using as input its own set of message/grade pairs. Informally, if a "relaxed" form of agreement on the input message/grade pairs has been established among the parties, this "relaxed" form of agreement still holds for the output message/grade pair. We make three observations regarding Combine. The first observation states that if one of the input messages is equal to $\perp$ and the corresponding grade is the maximum grade possible, then the output message will be equal to $\perp$ and the output grade will be the maximum grade possible.

Observation 1. If $m_{1}=\perp$ (resp., $\left.m_{2}=\perp\right)$ and $g_{1}=2^{d+1}+2^{d}-1$ (resp., $\left.g_{2}=2^{d+1}+2^{d}-1\right)$, then $m=\perp$ and $g=2^{d}+2^{d-1}-1$. 
The second observation is that if one of the input messages is equal to $m^{\prime} \notin\{\perp, \phi\}$, the corresponding grade is the maximum grade possible, and one of the three following conditions hold: (i) the other input message is equal to $\phi$; (ii) the other input grade is "low" (i.e., at most 1); or (iii) the two input messages are the same, then the output message will be equal to $m^{\prime}$ and the output grade will be the maximum grade possible.

Observation 2. If $m_{1} \notin\{\perp, \phi\} ; g_{1}=2^{d+1}+2^{d}-1$; and either (1) $m_{2}=\phi$ or (2) $g_{2} \leq 1$ or (3) $m_{2}=m_{1}$, then $m=m_{1}$ and $g=2^{d}+2^{d-1}-1$. Analogously, if $m_{2} \notin\{\perp, \phi\} ; g_{2}=2^{d+1}+2^{d}-1$; and either (1) $m_{1}=\phi$ or (2) $g_{1} \leq 1$ or (3) $m_{1}=m_{2}$, then $m=m_{2}$ and $g=2^{d}+2^{d-1}-1$.

The third observation is that if one of the input messages is equal to $\phi$, the corresponding grade is the maximum grade possible, and one of the two following conditions hold: (i) the other input message is equal to $\phi$ or (ii) the other input grade is "low" (i.e., at most 1), then the output message will be equal to $\phi$ and the output grade will be the maximum grade possible.

Observation 3. If $m_{1}=\phi ; g_{1}=2^{d+1}+2^{d}-1$; and either (1) $m_{2}=\phi$ or (2) $g_{2} \leq 1$, then $m=\phi$ and $g=2^{d}+2^{d-1}-1$. Analogously, if $m_{2}=\phi ; g_{2}=2^{d+1}+2^{d}-1$; and either (1) $m_{1}=\phi$ or (2) $g_{1} \leq 1$, then $m=\phi$ and $g=2^{d}+2^{d-1}-1$.

We are now ready to specify the second phase of the protocol. Recall that the parties in Init do not take part in this phase.

Protocol Rand-Bcast - Phase II:

Parties $P_{i} \in$ Rem perform the following steps:

1. For $1 \leq j \leq c+1$ set $m_{i, j}^{(\lceil\log (c+1)\rceil)}:=m_{i, j}$ and $g_{i, j}^{(\lceil\log (c+1)\rceil)}:=g_{i, j}$

for $c+2 \leq j \leq 2^{\lceil\log (c+1)\rceil}$ set $m_{i, j}^{(\lceil\log (c+1)\rceil)}:=\phi$ and $g_{i, j}^{(\lceil\log (c+1)\rceil)}:=0$.

2. For $d:=\lceil\log (c+1)\rceil$ to 1 do:

for $e:=1$ to $2^{d-1}$ do: $\left(m_{i, e}^{(d-1)}, g_{i, e}^{(d-1)}\right) \leftarrow$ Combine $\left(d, m_{i, 2 e-1}^{(d)}, g_{i, 2 e-1}^{(d)}, m_{i, 2 e}^{(d)}, g_{i, 2 e}^{(d)}\right)$.

3. Set $\left(m_{i}, g_{i}\right):=\left(m_{i, 1}^{(0)}, g_{i, 1}^{(0)}\right)$.

If $g_{i}=2$ then $P_{i}$ enters protocol $\mathrm{BA}_{\text {HonestMaj }}$ with input $m_{i}$, terminates $\mathrm{BA}_{\text {HonestMaj }}$ after $K$ rounds (where $K$ is the constant from Lemma 1), and outputs $m_{i}$.

else (i.e., $\left.g_{i}<2\right) P_{i}$ enters protocol $\mathrm{BA}_{\mathrm{HonestMaj}}$ with input $m_{i}$, runs $\mathrm{BA}_{\mathrm{HonestMaj}}$ until successful termination of the protocol, and outputs whatever directed to by $\mathrm{BA}_{\text {HonestMaj }}$.

We prove the following technical lemma in the Apendix which states that relaxed agreement is established on the message/grade pairs $\left\{\left(m_{i}, g_{i}\right)\right\}$.

Lemma 8. By the end of Phase II, the following holds for all honest parties $P_{i}, P_{j} \in \operatorname{Rem}$ :

- If $g_{i}>1$, then $m_{j}=m_{i}$ and $g_{j} \geq g_{i}-1$.

- If $g_{i}=1$, then $m_{j}=m_{i}$ or $g_{j}=0$.

We now argue that Rand-Bcast achieves broadcast. There are three cases: 
The sender $P_{1}$ is honest. By Lemma 3 , all honest parties in Init $\backslash\left\{P_{1}\right\}$ output $m$. For any honest party $P_{i} \in$ Rem, it follows from Lemma 3 and Observation 2 that $m_{i}=m$ and $g_{i}=2$ at the end of Phase II, which implies that $P_{i}$ outputs $m$.

$P_{1}$ is dishonest but there is an honest party $P_{i} \in \operatorname{Init} \backslash\left\{P_{1}\right\}$. Suppose $P_{i}$ outputs $\perp$. By Lemma 4, all honest parties in Init $\backslash\left\{P_{1}\right\}$ output $\perp$. Lemma 4 and Observation 1 show that, at the end of Phase II, $m_{j}=\perp$ and $g_{j}=2$ for any honest party $P_{j} \in$ Rem, which implies that $P_{j}$ outputs $\perp$. On the other hand, if $P_{i}$ outputs $\phi$ it follows from Lemma 5 and Observation 3 that all honest parties output $\phi$. Finally, if $P_{i}$ outputs $m \notin\{\perp, \phi\}$ it follows from Lemma 6 and Observation 2 that all honest parties output $m$.

All parties in Init are dishonest. This means that a strict majority of the parties in Rem are honest. There are two sub-cases. The first sub-case is that by the end of Phase II there exists an honest party $P_{i} \in$ Rem such that $g_{i}=2$. Then, by Lemma $8, m_{j}=m_{i}$ for all honest parties $P_{j}$ and so all honest parties will output the same value $m_{i}$. The second sub-case is that $g_{i} \leq 1$ for all honest parties $P_{i}$. In this case, it follows from the properties of $\mathrm{BA}_{\text {HonestMaj }}$ that all honest parties output the same message.

Phase I terminates in exactly $\mathcal{O}\left(k^{2}\right)$ rounds. Arguing as in the case of $t=n / 2$, we see that Phase II terminates in expected constant rounds. We thus obtain the following theorem:

Theorem 1. There exists an authenticated randomized n-party broadcast protocol tolerating $t=$ $n / 2+k$ dishonest parties that runs in (expected) $\mathcal{O}\left(k^{2}\right)$ rounds.

\section{A Lower Bound on the Round Complexity}

We start by considering a group of $k$ parties $P_{1}, P_{2}, \ldots, P_{k}$ such that only two of them are honest. We show that there does not exist any (randomized) broadcast protocol having any runs that terminate in fewer than $k-1$ rounds.

Consider a broadcast protocol $\Pi$ for $k$ parties that tolerates $k-2$ dishonest parties. For $1 \leq$ $i \leq k$, we construct a protocol $\bar{\Pi}_{i}$ that is the same as $\Pi$ except that:

- If $i=1$, then $P_{1}$ ignores all the messages sent to it except for those from $P_{2}$, and only sends messages to $P_{2}$ (i.e., $P_{1}$ only communicates with $P_{2}$ ).

- If $2 \leq i \leq k-1, P_{i}$ ignores all the messages sent to it except for those from $P_{i-1}$ and $P_{i+1}$, and only sends messages to $P_{i-1}$ and $P_{i+1}$ (i.e., $P_{i}$ only communicates with $P_{i-1}$ and $P_{i+1}$ ).

- If $i=k$, then $P_{k}$ ignores all the messages sent to it except for those from $P_{k-1}$, and only sends messages to $P_{k-1}$ (i.e., $P_{k}$ only communicates with $P_{k-1}$ ).

For $1 \leq i \leq k-1$ and $b \in\{0,1\}$, define scenario $S_{i}^{(b)}$ as follows:

- $P_{1}$ is the sender and the bit $b$ is its input.

- All parties except for $P_{i}$ and $P_{i+1}$ are dishonest. The honest parties $P_{i}$ and $P_{i+1}$ execute the protocol $\Pi$; a dishonest party $P_{j}$ executes the protocol $\bar{\Pi}_{j}$. 
For any $2 \leq i \leq k$, party $P_{i}$ cannot distinguish whether it is in $S_{i-1}^{(b)}$ or $S_{i}^{(b)}$. In scenario $S_{1}^{(b)}$, parties $P_{1}$ and $P_{2}$ are both honest. Thus, $P_{1}$ and $P_{2}$ have to output $b$ by the end of the protocol. Since $P_{2}$ cannot distinguish whether it is in $S_{1}^{(b)}$ or $S_{2}^{(b)}$, we see that $P_{2}$ has to output $b$ in scenario $S_{2}^{(b)}$ as well; this means that $P_{3}$ has to output $b$ as well. Prior to round 1 , however, the view of $P_{2}$ is completely independent of $b$, and so the view of $P_{3}$ is independent of $b$ prior to round 2 .

In general, in scenario $S_{i}^{(b)}$, parties $P_{i}$ and $P_{i+1}$ have to output $b$ and the view of $P_{i+1}$ is completely independent of $b$ prior to round $i$. If $b$ is chosen uniformly at random and $\Pi$ terminates before round $k-1$, then in scenario $S_{k-1}^{(b)}$ the output of $P_{k}$ will not be equal to $b$ with probability at least $1 / 2$. Since $\Pi$ is a broadcast protocol, $\Pi$ cannot terminate before round $k-1$. We conclude that there does not exist any broadcast protocol that can terminate in less than $k-1$ rounds if $k-2$ out of $k$ parties are dishonest.

Using standard player-partitioning techniques (see the Appendix), we can generalize the above to show:

Theorem 2. There does not exist any (randomized) n-party broadcast protocol tolerating $t$ dishonest parties that terminates in fewer than $2 n /(n-t)-1$ rounds (when $n-t \geq 2$ ).

\section{References}

[1] A. Bar-Noy, D. Dolev, C. Dwork, and H. R. Strong. Shifting gears: Changing algorithms on the fly to expedite Byzantine agreement. In 6th Annual ACM Symposium on Principles of Distributed Computing (PODC), 1987.

[2] M. Ben-Or. Another advantage of free choice: Completely asynchronous agreement protocols. In 2nd Annual ACM Symposium on Principles of Distributed Computing (PODC), 1983.

[3] M. Ben-Or, E. Pavlov, and V. Vaikuntanathan. Byzantine agreement in the full-information model in $O(\log n)$ rounds. In 38th Annual ACM Symposium on Theory of Computing (STOC), 2006.

[4] P. Berman and J. A. Garay. Efficient distributed consensus with $n=(3+\epsilon) t$ processors. In 5th Intl. Workshop on Distributed Algorithms (WDAG), 1991.

[5] P. Berman and J. A. Garay. Cloture votes: $n / 4$-resilient, polynomial-time distributed consensus in $t+1$ rounds. Mathematical Systems Theory, 26(1):3-20, 1993.

[6] C. Cachin, K. Kursawe, and V. Shoup. Random oracles in Constantitnople: Practical asynchronous Byzantine agreement using cryptography. In 19th Annual ACM Symposium on Principles of Distributed Computing (PODC), 2000.

[7] B. Chor and C. Dwork. Randomization in Byzantine agreement. Advances in Computing Research, 4, 1989. 
[8] R. Cleve. Limits on the security of coin flips when half the processors are faulty. In 18th Annual ACM Symposium on Theory of Computing (STOC), 1986.

[9] B. A. Coan. A communication-efficient canonical form for fault-tolerant distributed protocols. In 5th Annual ACM Symposium on Principles of Distributed Computing (PODC), 1986.

[10] J. Considine, M. Fitzi, M. Franklin, L. A. Levin, U. Maurer, and D. Metcalf. Byzantine agreement given partial broadcast. J. Cryptology, 18(3):191-217, 2005.

[11] D. Dolev. The Byzantine generals strike again. J. Algorithms, 3(1):14-30, 1982.

[12] D. Dolev, R. Reischuk, and H. R. Strong. Early stopping in Byzantine agreement. J. ACM, 37(4):720-741, 1990.

[13] D. Dolev and H. Strong. Authenticated algorithms for Byzantine agreement. SIAM J. Computing, 12(4):656-666, 1983.

[14] P. Feldman and S. Micali. An optimal probabilistic protocol for synchronous Byzantine agreement. SIAM J. Computing, 26(4):873-933, 1997.

[15] M. Fischer, N. Lynch, and M. Paterson. Impossibility of distributed consensus with one faulty processor. J. ACM, 32(2):374-382, 1985.

[16] M. J. Fischer and N. A. Lynch. A lower bound for the time to assure interactive consistency. Info. Proc. Lett., 14(4):183-186, 1982.

[17] M. Fitzi and J. A. Garay. Efficient player-optimal protocols for strong and differential consensus. In 22nd Annual ACM Symp. on Principles of Distributed Computing (PODC), 2003.

[18] J. A. Garay, P. D. MacKenzie, M. Prabhakaran, and K. Yang. Resource fairness and composability of cryptographic protocols. In 3rd Theory of Cryptography Conference (TCC), 2006.

[19] J. A. Garay and Y. Moses. Fully polynomial Byzantine agreement for $n>3 t$ processors in $t+1$ rounds. SIAM J. Computing, 27(1):247-290, 1998.

[20] S. Goldwasser and Y. Lindell. Secure multi-party computation without agreement. J. Cryptology, 18(3):247-287, 2005.

[21] S. Goldwasser, E. Pavlov, and V. Vaikuntanathan. Fault-tolerant distributed computing in full-information networks. In 47th Annual IEEE Symposium on Foundations of Computer Science (FOCS), 2006.

[22] R. Gradwohl, S. P. Vadhan, and D. Zuckerman. Random selection with an adversarial majority. In Advances in Cryptology - Crypto 2006.

[23] J. Katz and C.-Y. Koo. On expected constant-round protocols for Byzantine agreement. In Advances in Cryptology - Crypto 2006. 
[24] L. Lamport, R. Shostak, and M. Pease. The Byzantine generals problem. ACM Trans. Prog. Lang. Syst., 4(3):382-401, 1982.

[25] Y. Lindell, A. Lysyanskaya, and T. Rabin. On the composition of authenticated Byzantine agreement. In 34th Annual ACM Symposium on Theory of Computing (STOC), 2002.

[26] Y. Moses and O. Waarts. Coordinated traversal: $(t+1)$-round Byzantine agreement in polynomial time. J. Algorithms, 17(1):110-156, 1994.

[27] M. Naor and M. Yung. Universal one-way hash functions and their cryptographic applications. In 21st Annual ACM Symposium on Theory of Computing (STOC), 1989.

[28] J. Nielsen. A threshold pseudorandom function construction and its applications. In Advances in Cryptology - Crypto 2002.

[29] M. Pease, R. Shostak, and L. Lamport. Reaching agreement in the presence of faults. J. $A C M, 27(2): 228-234,1980$.

[30] B. Pfitzmann and M. Waidner. Information-theoretic pseudosignatures and Byzantine agreement for $t \geq n / 3$. Technical Report RZ 2882 (\#90830), IBM Research, 1996.

[31] M. Rabin. Randomized Byzantine generals. In 24th IEEE Symposium on Foundations of Computer Science (FOCS), 1983.

[32] J. Rompel. One-way functions are necessary and sufficient for secure signatures. In 22nd Annual ACM Symposium on Theory of Computing (STOC), 1990.

[33] S. Toueg, K. J. Perry, and T. K. Srikanth. Fast distributed agreement. SIAM J. Computing, 16(3):445-457, 1987.

\section{A Deferred Proofs}

\section{A.1 Correctness of M-Gradecast}

Lemma 2. Protocol M-Gradecast $\left(\cdot, g^{*}\right)$ is a $g^{*}$-gradecast protocol with round complexity $2 g^{*}+1$.

Proof. We first prove correctness. Suppose the sender is honest and let $P_{i}$ be any honest party. All parties receive $(m, \sigma)$ in round 1 . Since the adversary cannot forge signatures, $\left|S_{i}\right|=1$ and $m_{i}=m$ at all times. Hence $\bar{g}_{i}=2 g^{*}$ by the end of the protocol and $P_{i}$ will output $\left(m, g^{*}\right)$.

Next we prove soundness. Suppose there exists an honest party $P_{i}$ that outputs $g_{i} \geq 1$. Note that $\bar{g}_{i} \geq 2 g_{i}$ by the end of the protocol. Let round $r_{1}$ be the round during which $m_{i}$ is added to $S_{i}$ by $P_{i}$. Then $\left|S_{i}\right|=0$ (and hence $\bar{g}_{i}=0$ ) prior to round $r_{1}$. We claim that if there exists an honest party $P_{j}$ who receives $\left(m^{\prime}, \sigma^{\prime}\right)$ in round $r_{2}$ such that $m^{\prime} \neq m_{i}$ and $\sigma^{\prime}$ is a valid signature on $m$ by the sender, then $r_{2}>r_{1}+2 g_{i}-3$. Assume the claim is not true, i.e., $r_{2} \leq r_{1}+2 g_{i}-3$. Since $P_{j}$ is honest, it sends $\left(m^{\prime}, \sigma^{\prime}\right)$ to all parties (including $\left.P_{i}\right)$ in round $r_{2}+1$. Then by the end of step (a) 
in round $r_{2}+2$, it holds that $\left|S_{i}\right| \geq 2$ (note that $S_{i}$ contains $m_{i}$ by then as $m_{i}$ is the first message added to it). Hence the value of $\bar{g}_{i}$ is at most $r_{2}+2-r_{1} \leq 2 g_{i}-1$, a contradiction.

We now complete the proof. Since $P_{i}$ is honest, $P_{i}$ sends $m_{i}$ along with a valid signature from the sender to all parties in round $r_{1}$. All honest parties receive it by the end of round $r_{1}$. The claim we proved in the last paragraph states that no honest party $P_{j}$ receives a different message $m^{\prime} \neq m_{i}$ (with a valid signature from the sender) in or before round $r_{1}+2 g_{i}-3$. Consider the value of $\bar{g}_{j}$ by the end of the protocol. If $g_{i} \geq 2$, then $\bar{g}_{j} \geq r_{1}+2 g_{i}-3+1-r_{1} \geq 2 g_{i}-2$, and so $P_{j}$ outputs $m_{i}$ with $g_{j} \geq g_{i}-1$. For the case $g_{i}=1$, following the claim in the previous paragraph, no honest party $P_{j}$ receives a message $m^{\prime}$ different from $m_{i}$ (with a valid signature from the sender) in or before round $r_{1}-1$. Since $P_{j}$ receives $m_{i}$ (along with a valid signature from the sender) in round $r_{1}$, it holds that $m_{i} \in S_{j}$ by the end of step (a) in round $r_{1}+1$. It follows that $g_{j}=0$ (if a different message $m^{\prime}$ is received by $P_{j}$ in round $r_{1}$ ) or $m_{j}=m_{i}$.

\section{A.2 Properties of Rand-Bcast}

Lemma 3. If the sender $P_{1}$ is honest, the following holds at the end of Phase I:

1. All honest parties in Init $\backslash\left\{P_{1}\right\}$ output $m$;

2. For all honest parties $P_{i} \in$ Rem, it holds that $m_{i, 1}=m$ and $g_{i, 1}=g^{*}$. Furthermore, for each $2 \leq j \leq c+1$ it holds that $m_{i, j}=m$ or $m_{i, j}=\phi$ (this holds even if $P_{j}$ is dishonest).

Proof. If the sender $P_{1}$ is honest, then in step 1 all honest parties $P_{i} \in \ln i t \backslash\left\{P_{1}\right\}$ receive $\left(m, \sigma, P_{1}\right)$ as the output of the gradecast by $P_{1}$, where $\sigma$ is a valid signature on $m$ by $P_{1}$. Hence $m \in M_{i}$ by the end of step 2. Since the adversary cannot forge a signature of $P_{1}$, no message besides $m$ will be added to $M_{i}$ by the end of Phase I. Thus, all honest parties $P_{i} \in \operatorname{Init} \backslash\left\{P_{1}\right\}$ will output $m$.

For all honest parties $P_{j} \in$ Rem, we have $m_{j, 1}=m$ and $g_{j, 1}=g^{*}$ by the properties of M-Gradecast. Furthermore, $m_{j, i}=m$ or $m_{j, i}=\phi$ for any $2 \leq i \leq c+1$ as the adversary cannot forge a valid signature of $P_{1}$.

We prove two technical results that will be used in the proofs of Lemmas 4-6.

Lemma 9. Let $P_{i} \in \operatorname{Init} \backslash\left\{P_{1}\right\}$ be honest. If $m \in M_{i}$ by the end of Phase I, then:

1. For any honest $P_{j} \in \operatorname{Init} \backslash\left\{P_{1}\right\}$ it holds that $m \in M_{j}$ or $\left|M_{j}\right|=2$.

2. For any honest $P_{j} \in \operatorname{Rem}$ it holds that $m \in M_{j, i}$.

Proof. Suppose $m$ is added to $M_{i}$ in step $k$. Then in step $k-1$, party $P_{i}$ received a message $m_{i, \alpha_{k-2}}^{\prime}=\left(m, \sigma_{\alpha_{0}}, P_{1}, \sigma_{\alpha_{1}}, P_{\alpha_{1}}, \ldots, \sigma_{\alpha_{k-2}}, P_{\alpha_{k-2}}\right)$ as the output of a gradecast by some party $P_{\alpha_{k-2}}$. In step $k, P_{i}$ verifies that $m_{i, \alpha_{k-2}}^{\prime}$ is valid, adds $m$ to $M_{i}$, computes a signature $\sigma_{\alpha_{j-1}}$ of $\sigma_{\alpha_{j-2}}$, and gradecasts $\left(m_{\alpha_{k-2}, i}^{\prime}, \sigma_{\alpha_{j-1}}, P_{i}\right)$. All honest parties receive $\left(m_{i, \alpha_{k-2}}^{\prime}, \sigma_{\alpha_{j-1}}, P_{i}\right)$ as the output of that gradecast. Since $m$ is added to $M_{i}$ in step $k$, it means that $m$ is not in $M_{i}$ in step $k-1$. Therefore, $P_{i} \notin\left\{P_{1}, P_{\alpha_{1}}, \ldots, P_{\alpha_{k-2}}\right\}$. This implies that $k \leq c+1$.

We know that $\left(m_{i, \alpha_{k-2}}^{\prime}, \sigma_{\alpha_{j-1}}, P_{i}\right)$ is valid in step $k+1$. Consider an honest $P_{j} \in \operatorname{lnit} \backslash\left\{P_{1}\right\}$. If $m$ is not added to $M_{j}$ in step $k+1$, then it means that $m$ is already in $M_{j}$ or $\left|M_{j}\right|=2$ by the end of step $k+1$. This proves the first item. Next consider an honest party $P_{j} \in$ Rem. Following the 
properties of M-Gradecast and the protocol description, $m \in M_{j, i}$ by the end of step $k+1$, which proves the second item.

Lemma 10. Let $P_{i} \in$ Rem be honest. If, for some $P_{j} \in$ Init, it holds that $m \in M_{i, j}$ and $g_{i, j} \geq 2$ at the end of Phase I, then for all honest parties $P_{k} \in \operatorname{Init} \backslash\left\{P_{1}\right\}$ it holds that either $m \in M_{k}$ or $\left|M_{k}\right|=2$ at the end of Phase $I$.

Proof. Suppose $m$ is added to $M_{i, j}$ in step $r$. This means $P_{j}$ gradecasts $m_{j}=\left(m, \sigma_{\alpha_{0}}, \ldots, \sigma_{\alpha_{r-2}}, P_{j}\right)$ in step $r-1$, and $P_{i}$ receives $m_{j}$ with grade at least 2. Following the properties of M-Gradecast, all honest parties receive $m_{j}$ with grade at least 1 . We know that $m_{j}$ is valid in step $r$ since $m$ is added to $M_{i, j}$ in step $r$. Therefore, by the end of step $r$, it holds that $m \in M_{k}$ or $\left|M_{k}\right|=2$ for all honest parties $P_{k} \in \operatorname{Init} \backslash\left\{P_{1}\right\}$.

Lemma 4. If any honest party $P_{i} \in$ Init $\backslash\left\{P_{1}\right\}$ outputs $\perp$, then all honest parties in Init $\backslash\left\{P_{1}\right\}$ output $\perp$, and for any honest $P_{j} \in \operatorname{Rem}$ it holds that $m_{j, i}=\perp$ and $g_{j, i}=g^{*}$ at the end of Phase I.

Proof. If $P_{i}$ outputs $\perp$, then $\left|M_{i}\right|=2$ by the end of Phase I. Using Lemma 9, by the end of Phase I $\left|M_{j}\right|=2$ for all honest parties $P_{j} \in$ Init $\backslash\left\{P_{1}\right\}$. Therefore $P_{j}$ outputs $\perp$. If $P_{j} \in$ Rem is honest, $P_{j}$ always receives grade $g^{*}$ in every gradecast by $P_{i}$. By Lemma $9, m_{j, i}=\perp$ and $g_{j, i}=g^{*}$.

We prove Lemma 6 first, since we rely on it to prove Lemma 5.

Lemma 6. If any honest party $P_{i} \in$ Init $\backslash\left\{P_{1}\right\}$ outputs $m \notin\{\perp, \phi\}$, then all honest parties in Init $\backslash\left\{P_{1}\right\}$ output $m$, and for any honest $P_{j} \in \operatorname{Rem}$ it holds that $m_{j, i}=m$ and $g_{j, i}=g^{*}$ at the end of Phase I. Moreover, if $m_{j, k} \neq m$ and $m_{j, k} \neq \phi$ for some $k \in\{1, \ldots, c+1\}$, then $g_{j, k} \leq 1$.

Proof. By the end of Phase I, $m \in M_{i}$. Consider an honest party $P_{j} \in \operatorname{Init} \backslash\left\{P_{1}\right\}$. By Lemma 9, we have $m \in M_{j}$ by the end of Phase I. If $P_{j}$ does not output $m$, then $\left|M_{j}\right|=2$ which means $P_{j}$ outputs $\perp$. By Lemma $4, P_{i}$ should output $\perp$ instead of $m$, a contradiction.

Next consider an honest party $P_{j} \in$ Rem. We know that $m_{j, i}=m$ and $g_{j, i}=g^{*}$ by the properties of M-Gradecast. Now suppose there exists a $1 \leq k \leq c+1$ such that $m_{j, k} \neq m$ and $m_{j, k} \neq \phi$. Then there exists $m^{\prime} \neq m$ such that $m^{\prime} \in M_{j, k}$ by the end of Phase I. By Lemma 10, this means $g_{j, k} \leq 1$ or $m^{\prime} \in M_{i}$ or $\left|M_{i}\right|=2$ by the end of Phase I. Since $P_{i}$ outputs $m$, we have $M_{i}=\{m\}$ and this means $g_{j, k} \leq 1$.

Lemma 5. If any honest party $P_{i} \in \operatorname{Init} \backslash\left\{P_{1}\right\}$ outputs $\phi$, then all honest parties in Init $\backslash\left\{P_{1}\right\}$ output $\phi$, and for any honest $P_{j} \in \operatorname{Rem}$ it holds that $m_{j, i}=\phi$ and $g_{j, i}=g^{*}$ at the end of Phase I. Moreover, if $m_{j, k} \neq \phi$ for some $k \in\{1, \ldots, c+1\}$, then $g_{j, k} \leq 1$.

Proof. Consider an honest party $P_{j} \in$ Init $\backslash\left\{P_{1}\right\}$. If $P_{j}$ does not output $\phi$ then, using Lemma 4 and Lemma $6, P_{i}$ should output $\perp$ or $m^{\prime}$ instead, a contradiction.

Now consider an honest party $P_{j} \in$ Rem. Properties of M-Gradecast imply that $m_{j, i}=\phi$ and $g_{j, i}=g^{*}$. Suppose there exists a $1 \leq k \leq c+1$ such that $m_{j, k} \neq \phi$. Then there exists an $m^{\prime} \in M_{j, k}$ by the end of Phase I. Following Lemma 10, $g_{j, k} \leq 1$ or $m^{\prime} \in M_{i}$ or $\left|M_{i}\right|=2$. Since $P_{i}$ outputs $\phi$, this implies that $g_{j, k} \leq 1$. 
Lemma 8. By the end of Phase II, the following holds for all honest parties $P_{i}, P_{j} \in$ Rem:

- If $g_{i}>1$, then $m_{j}=m_{i}$ and $g_{j} \geq g_{i}-1$.

- If $g_{i}=1$, then $m_{j}=m_{i}$ or $g_{j}=0$.

Proof. The lemma follows once we show that, by the end of Phase II, for any $0 \leq d \leq\lceil\log (c+1)\rceil$ and $1 \leq e \leq 2^{d}$ :

- If $g_{i, e}^{(d)}>1$ for some honest party $P_{i} \in$ Rem, then $m_{j, e}^{(d)}=m_{i, e}^{(d)}$ and $g_{j, e}^{(d)} \geq g_{i, e}^{(d)}-1$ for any honest party $P_{j} \in$ Rem .

- If $g_{i, e}^{(d)}=1$ for some honest party $P_{i} \in \operatorname{Rem}$, then either $m_{j, e}^{(d)}=m_{i, e}^{(d)}$ or $g_{j, e}^{(d)}=0$ for any honest party $P_{j} \in$ Rem.

We prove the above by induction on $d$.

Base Case: The statement is true for $d=\lceil\log (c+1)\rceil$ and any $e$ by Lemma 7 .

Inductive Step: Assume the statement is true for $d=d^{\prime}+1$ and $e=2 e^{\prime}-1$ and $e=2 e^{\prime}$. We show that the statement is true for $d=d^{\prime}$ and $e=e^{\prime}$. We have the following cases:

1. Suppose that for all honest parties $P_{i}, P_{j} \in$ Rem, we have $m_{i, 2 e^{\prime}-1}^{\left(d^{\prime}+1\right)}=m_{j, 2 e^{\prime}-1}^{\left(d^{\prime}+1\right)}$. Consider the two sub-cases:

- $m_{i, 2 e^{\prime}}^{\left(d^{\prime}+1\right)}=m_{j, 2 e^{\prime}}^{\left(d^{\prime}+1\right)}$ for all honest parties $P_{i}, P_{j}$. Then following the protocol specification, the statement is true for $d=d^{\prime}$ and $e=e^{\prime}$.

- $m_{i, 2 e^{\prime}}^{\left(d^{\prime}+1\right)} \neq m_{j, 2 e^{\prime}}^{\left(d^{\prime}+1\right)}$ for some honest parties $P_{i}, P_{j}$. This means $g_{k, 2 e^{\prime}}^{\left(d^{\prime}+1\right)} \leq 1$ for all honest parties $P_{k}$. Following the protocol specification, if $g_{k, 2 e^{\prime}-1}^{\left(d^{\prime}+1\right)}>2^{d}+2^{d-1}$, then $m_{k, e^{\prime}}^{\left(d^{\prime}\right)}=m_{k, 2 e^{\prime}-1}^{\left(d^{\prime}+1\right)}$ and $g_{k, e^{\prime}}^{\left(d^{\prime}\right)}=g_{k, 2 e^{\prime}-1}^{\left(d^{\prime}+1\right)}-2^{d}-2^{d-1}$, else $g_{k, e^{\prime}}^{\left(d^{\prime}\right)}=0$. Thus the statement is true for $d=d^{\prime}$ and $e=e^{\prime}$.

2. Next suppose that for all honest parties $P_{i}, P_{j} \in$ Rem, it holds that $m_{i, 2 e^{\prime}}^{\left(d^{\prime}+1\right)}=m_{j, 2 e^{\prime}}^{\left(d^{\prime}+1\right)}$. The proof of this case is analogous to the previous case.

3. Finally, consider the case where neither condition above holds. This means that $g_{i, 2 e^{\prime}-1}^{\left(d^{\prime}+1\right)} \leq 1$ and $g_{i, 2 e^{\prime}}^{\left(d^{\prime}+1\right)} \leq 1$ for all honest parties $P_{i}$. Following the protocol specification, $g_{i, e^{\prime}}^{\left(d^{\prime}\right)}=0$. Hence the statement holds.

\section{A.3 The Lower Bound}

Theorem 2. There does not exist any (randomized) n-party broadcast protocol tolerating $t$ dishonest parties that terminates in fewer than $2 n /(n-t)-1$ rounds (when $n-t \geq 2$ ).

Proof. Let $h=n-t$. We divide the parties into $k=n /(h / 2)$ disjoint groups $G_{1}, \ldots, G_{k}$, each of size $h / 2$. Consider a broadcast protocol $\Pi$ for $n$ parties that can tolerate $t$ dishonest parties. For $1 \leq i \leq k$, we construct a protocol $\bar{\Pi}_{i}$ that is the same as $\Pi$ except that 
- If $i=1$, then the parties in $G_{1}$ ignore all the messages sent to them except for those from the parties in $G_{1} \cup G_{2}$, and only send messages to the parties in $G_{1} \cup G_{2}$ (i.e., parties in $G_{1}$ only communicates with parties in $G_{1} \cup G_{2}$ ).

- If $2 \leq i \leq k-1$, parties in $G_{i}$ ignore all the messages sent to them except for those from the parties in $G_{i-1} \cup G_{i} \cup G_{i+1}$, and only send messages to parties in $G_{i-1} \cup G_{i} \cup G_{i+1}$ (i.e., parties in $G_{i}$ only communicates with parties in $G_{i-1} \cup G_{i} \cup G_{i+1}$ ).

- If $i=k$, then the parties in $G_{k}$ ignore all the messages sent to them except for those from the parties in $G_{k-1} \cup G_{k}$, and only send messages to the parties in $G_{k-1} \cup G_{k}$ (i.e., parties in $G_{k}$ only communicates with parties in $G_{k-1} \cup G_{k}$ ).

For $1 \leq i \leq k-1$ and $b \in\{0,1\}$, define scenario $S_{i}^{(b)}$ as follows:

- The sender is in $G_{1}$ and the bit $b$ is its input.

- All parties except for the parties in $G_{i} \cup G_{i+1}$ are dishonest.

- The honest parties in $G_{i} \cup G_{i+1}$ execute protocol $\Pi$; each dishonest party in $G_{j}$ executes protocol $\bar{\Pi}_{j}$.

The rest of the proof proceeds analogously to the discussion in Section 4. 